\title{
A Note on Differentiability of Lipschitz Maps
}

by

\author{
Rafał GÓRAK
}

\author{
Presented by Aleksander PEŁCZYŃSKI
}

\begin{abstract}
Summary. We show that every Lipschitz map defined on an open subset of the Banach space $C(K)$, where $K$ is a scattered compactum, with values in a Banach space with the Radon-Nikodym property, has a point of Fréchet differentiability. This is a strengthening of the result of Lindenstrauss and Preiss who proved that for countable compacta. As a consequence of the above and a result of Arvanitakis we prove that Lipschitz functions on certain function spaces are Gâteaux differentiable.
\end{abstract}

1. Introduction. We investigate the problem of differentiation of Lipschitz functions on Banach spaces. Results concerning differentiation provide an important technique of linearization of nonlinear maps and therefore many problems in nonlinear classification of Banach spaces can be reduced to the classical linear theory.

Let us recall some basic definitions and facts:

Definition 1.1. Let $f: U \rightarrow F$ be a Lipschitz map where $F$ is a Banach space and $U \neq \emptyset$ is an open set of a Banach space $E$. We say that $f$ is Gâteaux differentiable at $x_{0} \in U$ if there exists a continuous linear operator $D f\left(x_{0}\right): E \rightarrow F$ such that

$$
D f\left(x_{0}\right) u=\lim _{t \rightarrow 0}\left(f\left(x_{0}+t u\right)-f\left(x_{0}\right)\right) / t \quad \text { for all } u \in E .
$$

Moreover, if the above limit exists uniformly in $u$ on the unit sphere in $E$ then we say that $f$ is Fréchet differentiable at $x_{0}$. Let us denote by $\operatorname{Diff}_{\mathrm{F}}(f)$ and $\operatorname{Diff}_{G}(f)$ the sets of all points of Fréchet and Gâteaux differentiability of $f$, respectively.

2010 Mathematics Subject Classification: 46G05, 58C20, 54G12.

Key words and phrases: Lipschitz map, Gâteaux and Fréchet differentiability, scattered spaces. 
Obviously if $E$ is finite-dimensional and the function $f$ is Lipschitz then the notions of Fréchet and Gâteaux differentiability coincide.

Let us recall the classical fact about differentiability of Lipschitz functions:

THEOREM 1.2 (Rademacher). Let $f: \mathbb{R}^{n} \rightarrow \mathbb{R}^{k}$ be a Lipschitz function. Then the set $\operatorname{Diff}_{\mathrm{F}}(f)$ is of full Lebesgue measure in $\mathbb{R}^{n}$.

We need some definitions before quoting more recent results:

Definition 1.3. We say that $F$ has the Radon-Nikodym property $(F \in$ RNP) if every Lipschitz function $f: \mathbb{R} \rightarrow F$ is differentiable almost everywhere (equivalently, at some point).

Typical examples of Banach spaces having the Radon-Nikodym property are all reflexive Banach spaces. It is also worth mentioning that spaces having RNP can be defined differently in the spirit of the Radon-Nikodym theorem (see [6] for more information).

The next definition gives a necessary condition that the codomain must satisfy in order that most of the relevant differentiability results work.

Definition 1.4. We say that a Banach space $F$ is Asplund if for every closed separable subspace $X \subset F$ the space $X^{*}$ is separable.

Theorem 1.5 (Aronszajn [2], Christensen [8], Mankiewicz [14]). Let E be a separable Banach space and $F \in R N P$. Then every Lipschitz map $f$ : $U \rightarrow F$, where $U \neq \emptyset$ is an open subset of $E$, is Gâteaux differentiable outside a set $I$ belonging to some non-trivial sigma ideal $\Sigma \varsubsetneqq \mathcal{P}(U)$.

In the papers of Aronszajn, Christensen and Mankiewicz the above theorem was proved for different sigma ideals $\Sigma$. Let us mention that Lindenstrauss and Preiss [13] recently defined another sigma ideal of gamma null sets for which the conclusion of Theorem 1.5 holds. Let us denote this sigma ideal by $\Gamma$. Unfortunately the above theorem is no longer true if we consider Fréchet differentiability. Define $\varphi: \ell_{2} \rightarrow \ell_{2}$ by $\varphi\left(\left(x_{i}\right)_{i \in \mathbb{N}}\right)=\left(\left|x_{i}\right|\right)_{i \in \mathbb{N}}$. It is not difficult to check that $\varphi$ is a Lipschitz map which is nowhere Fréchet differentiable. However if the codomain is the space of real numbers $\mathbb{R}$ then we have the following theorem:

Theorem 1.6 (Preiss [17]). Let $U \neq \emptyset$ be an open subset of an Asplund space and $f: U \rightarrow \mathbb{R}$ be a Lipschitz map. Then $\operatorname{Diff}_{\mathrm{F}}(f)$ is dense in $U$.

The assumption that $U$ is a subset of an Asplund space cannot be weakened. Namely, if $E$ is not an Asplund space then there exists an equivalent norm on $E$ which is nowhere Fréchet differentiable (see Corollary 2.35 of [16]). It is also interesting when the space $\mathbb{R}$ can be replaced by any Banach space having RNP. For instance the map $f: \ell_{2} \rightarrow \ell_{2}$ defined by 
$f\left(\left(x_{1}, x_{2}, \ldots\right)\right)=\left(\left|x_{1}\right|,\left|x_{2}\right|, \ldots\right)$ can be shown to be nowhere Fréchet differentiable. However in some special cases this can be done:

Theorem 1.7 (Lindenstrauss and Preiss [13]). Let $M$ be a countable compact space and $F$ be a Banach space having RNP. Then every Lipschitz map $f: U \rightarrow F$ defined on an open subset $U \subset C(M)$ is Fréchet differentiable outside a set $I \in \Gamma \subset \mathcal{P}(U)$.

Here and below, $C(M)$ denotes the Banach space of all real valued continuous functions on $M$ equipped with the supremum norm.

Before we finish the introduction let us formulate a simple fact concerning Gâteaux differentiability. We use it in the last section of this article.

FACT 1.8. Let $V$ and $W$ be two Banach spaces and $T: V \rightarrow W$ be a continuous linear operator such that $\overline{T V}=W$. Let $f: W \rightarrow Y$ be a Lipschitz function where $Y$ is a Banach space. Then $T\left(\operatorname{Diff}_{\mathrm{G}}(f \circ T)\right) \subset \operatorname{Diff}_{\mathrm{G}}(f)$.

Proof. We need the following simple fact (see Lemma 6.40 in [6]):

Lemma 1.9. Let $E$ and $F$ be Banach spaces, and let $f$ be a Lipschitz function from an open set in $E$ into $F$. Let $G$ be a dense additive subgroup of $E$, and assume that for some $x_{0} \in E$ and for all $u \in G$ the directional derivative at $x_{0}$

$$
\lim _{t \rightarrow 0} \frac{f\left(x_{0}+t u\right)-f\left(x_{0}\right)}{t}
$$

exists and is additive as a function of $u$. Then $f$ is Gâteux differentiable at $x_{0}$.

Take $x_{0} \in \operatorname{Diff}_{\mathrm{G}}(f \circ T)$. From the linearity of $T$ we know that the limit

$$
\lim _{t \rightarrow 0} \frac{f\left(T x_{0}+t T v\right)-f\left(T x_{0}\right)}{t}
$$

exists for all $v \in V$ and is additive as a function of $T v$. Lemma 1.9 and the obvious fact that $T V$ is a dense additive subgroup of $W$ finish the proof of Fact 1.8

2. Fréchet differentiability of Lipschitz maps on function spaces. Let us recall that the space $K$ is scattered if every nonempty subset of $K$ has an isolated point. Obviously all countable compact spaces are scattered. This section will be devoted to a generalization of Theorem 1.7. Precisely, we will prove that:

THEOREM 2.1. Let $K$ be a compact scattered space, $G$ an open subset of $C(K)$, and $Y$ a Banach space having RNP. Then every Lipschitz map $f: G \rightarrow Y$ has a point of Fréchet differentiability.

An immediate corollary of the above theorem is 
Corollary 2.2. Let $K$ be a compact scattered space, $G$ an open subset of $C(K)$, and $\left\{Y_{n}\right\}_{n \in \mathbb{N}}$ Banach spaces having $R N P$. Then for every sequence of Lipschitz maps $f_{n}: G \rightarrow Y_{n}$ we have $\bigcap_{n=1}^{\infty} \operatorname{Diff}_{\mathrm{F}}\left(f_{n}\right) \neq \emptyset$.

Proof. Without loss of generality we can assume that the Lipschitz constants of all the $f_{n}$ are 1 . Define $g: G \rightarrow Y=\left(Y_{1} \times Y_{2} \times \cdots\right)_{\ell_{1}}$ by $g(x)=$ $\left(f_{1}(x) / 1^{2}, f_{2}(x) / 2^{2}, f_{3}(x) / 3^{2}, \ldots\right)$. Since $Y$ has RNP, from Theorem 2.1 we know that there exists $x_{0} \in G$ which is a point of Fréchet differentiability of $g$. It is easy to check that $x_{0} \in \bigcap_{n=1}^{\infty} \operatorname{Diff}_{\mathrm{F}}\left(f_{n}\right)$, which finishes the proof.

Let us note that the assumption on $K$ cannot be weakened because of the following classical fact:

FACT 2.3. The Banach space $C(K)$ is an Asplund space if and only if $K$ is scattered.

The proof of this fact can be found in [10]. We will reduce Theorem 2.1 to the separable case (i.e. to Theorem 1.7). We need the following notion which appears in [7].

Definition 2.4. Let $E$ be a normed linear space. We call a family $\mathcal{F}$ of closed separable subspaces of $E$ rich if the following conditions are satisfied:

(i) If $\left\{V_{n}\right\}_{n \in \mathbb{N}} \subset \mathcal{F}$ is an increasing family then $\overline{\bigcup\left\{V_{n} ; n \in \mathbb{N}\right\}} \in \mathcal{F}$.

(ii) For every separable subspace $V \subset E$ there exists $W \in \mathcal{F}$ such that $V \subset W$.

The next theorem is essentially due to Preiss (see [17]).

TheOrem 2.5. Let $f: G \rightarrow F$ be a continuous map of an open subset $G \subset E$ into $F$ where $E$ and $F$ are Banach spaces, and let $\mathcal{F}$ be a rich family of subspaces of $E$. Then for every separable subspace $V \subset E$ there exists $W \in \mathcal{F}$ such that $V \subset W$ and

(*) $\operatorname{Diff}_{\mathrm{F}}(f \mid W \cap G)=W \cap G \cap \operatorname{Diff}_{\mathrm{F}}(f)$.

Proof. For completeness we provide an idea of the proof. For every $x \in G$ let $A(x) \subset S_{E}$ be a countable subset of the unit sphere such that for all $s, t \neq 0$ satisfying $\max \{|s|, 2|t|\}<\operatorname{dist}(x, E \backslash G)$ there exist $u, v \in A(x)$ such that:

$$
\begin{aligned}
\| \frac{f(x+t(u+v))-}{t} & f(x) \\
\geq & -\frac{f(x+s u)-f(x)}{s}-\frac{f(x+s v)-f(x)}{s} \| \\
& \left.\quad-\frac{f(x+s z)-f(x)}{s}-\frac{f(x+s w)-f(x)}{s} \|: z, w \in S_{E}\right\} .
\end{aligned}
$$


The existence of such a set follows from the fact that it is enough to consider $s, t \in \mathbb{Q} \backslash\{0\}$ only.

We define inductively a sequence of subspaces $\left\{V_{n}\right\}_{n \in \mathbb{N}}$ where $V_{0}=V$ and $V_{n+1}$ is the element of $\mathcal{F}$ containing $\overline{\operatorname{span}}\left(C_{n} \cup A\left(C_{n} \cap G\right)\right)$ where $C_{n}$ is a dense and countable subspace of $V_{n}$. In his paper Preiss shows that the desired $W \in \mathcal{F}$ can be defined as $W=\overline{\bigcup\left\{V_{n} ; n \in \mathbb{N}\right\}}$. He considers only the case when the family $\mathcal{F}$ consists of all closed and separable subspaces of $E$. However the proof remains the same for any rich family $\mathcal{F}$. The only difference is the definition of $\left\{V_{n}\right\}_{n \in \mathbb{N}}$. Since all the remaining part of the proof is the same as in [17], we do not provide further details.

Proof of Theorem 2.1. It is clear that the family $\mathcal{F}$ of all closed and separable subalgebras of $C(K)$ is rich. On the other hand if $A \in \mathcal{F}$ then $A$ is isometric to $C(L)$ for some compactum $L$ (Gelfand-Naimark theorem). Therefore $C(L)$ embeds linearly and isometrically into $C(K)$. Holsztyński's theorem [12] shows that $L$ is a continuous image of a subset of $K$, hence it is scattered. Since $C(L)$ is separable, the compactum $L$ is of countable weight, hence metrizable. Thus $L$, being a metrizable and scattered compact space, must be countable. Theorems 1.7 and 2.5 finish the proof.

3. Gâteaux differentiability and scattered generated spaces. In this section we shall show how Theorem 2.1 yields results concerning Gâteaux differentiability of Lipschitz maps on nonseparable Banach spaces with values in RNP spaces. It turns out that the results obtained are connected with the branch of topology dealing with different kinds of weakly compact spaces such as Eberlein or Radon-Nikodym compacta. For more information on this topic see [5] and [10].

Definition 3.1. Let $E$ and $F$ be Banach spaces and $T: E \rightarrow F$ be a continuous linear operator. We say that $E$ generates $F$ if $\overline{T E}=F$. When additionally $E=C(K)$ for $K$ scattered, we call $F$ a scattered generated space.

As an immediate corollary of Theorem 2.1 and Fact 1.8 we obtain the following:

COROLlARY 3.2. Every countable family of Lipschitz maps on a scattered generated space $F$ with values in a space with RNP has a common point of Gâteaux differentiability.

Therefore a natural question arises:

QUESTION 3.3. Which Banach spaces are scattered generated?

First of all note that all separable Banach spaces are scattered generated. Indeed, all of them are generated by $\ell_{2}$ (cf. [9], Lemma 2.5, p. 47]), hence 
also by $c_{0}$. However this is no longer true if we consider all Hilbert spaces, as the following well known fact shows:

FACT 3.4. Let $f: \ell_{2}(\Gamma) \rightarrow \ell_{2}(\Gamma)$ be defined by $f\left(\left\{x_{\gamma}\right\}_{\gamma \in \Gamma}\right)=\left\{\left|x_{\gamma}\right|\right\}_{\gamma \in \Gamma}$. For all uncountable $\Gamma$ we have $\operatorname{Diff}_{G}(f)=\emptyset$. In particular $\ell_{2}(\Gamma)$ is not scattered generated.

Let us list some obvious properties of the class of scattered generated spaces:

FACT 3.5. Let $\left\{E_{i}\right\}_{i \in \mathbb{N}}$ be scattered generated spaces. Then the following spaces are scattered generated:

(i) $E_{1} \times E_{2}$,

(ii) $\left(E_{1} \times E_{2} \times \cdots\right)_{c_{0}}$ and $\left(E_{1} \times E_{2} \times \cdots\right)_{\ell_{p}}$ for $p \geq 1$.

Proof. Observe that $\left(E_{1} \times E_{2} \times \cdots\right)_{c_{0}}$ generates $\left(E_{1} \times E_{2} \times \cdots\right)_{\ell_{p}}$ for $p \geq 1$. Let $K_{n}(n \in \mathbb{N})$ be scattered spaces such that $C\left(K_{n}\right)$ generates $E_{n}$. Since $\left(C\left(K_{1}\right) \times C\left(K_{2}\right) \times \cdots\right)_{c_{0}}$ is isomorphic to $C(K)$ where $K$ is the one-point compactification of the disjoint sum of the scattered spaces $K_{1}, K_{2}, \ldots$, we find that $\left(E_{1} \times E_{2} \times \cdots\right)_{c_{0}}$ is generated by $C(K)$. Obviously $K$ is scattered itself.

In the following considerations we shall focus on scattered generated $C(L)$ spaces. Let us introduce the following definition:

Definition 3.6. A compact space $L$ belongs to the class $\mathcal{G}$ if $C(L)$ is scattered generated.

Let us now define some known classes of compact spaces that in the end will turn out to be subclasses of $\mathcal{G}$.

Definition 3.7. Let $L$ be a compact space. We call $L$

(i) a uniformly Eberlein compactum if $L$ is homeomorphic to a weakly compact space of a Hilbert space,

(ii) an Eberlein compactum if $L$ is homeomorphic to a weakly compact space of a Banach space,

(iii) an almost totally disconnected space if there exists a homeomorphic embedding $\varphi$ of $L$ into a Hilbert cube $[0,1]^{\Gamma}$ such that for every $l \in L$ all but countably many coordinates of $\varphi(l) \in[0,1]^{\Gamma}$ are 0 or 1 .

It is easy to notice that uniformly Eberlein compacta are Eberlein. On the other hand the characterization of Amir and Lindenstrauss in 1 1 says that Eberlein compacta are weakly compact subsets of $c_{0}(\Gamma)$, which implies that the class of almost totally disconnected compact spaces contains all Eberlein compacta. 
To see that the classes defined in Definition 3.7 are subclasses of $\mathcal{G}$ we need the following dual characterization of $\mathcal{G}$ :

FACT 3.8. A compact space $L$ belongs to $\mathcal{G}$ if and only if it can be embedded into $\left(B_{C(K)^{*}}, w^{*}\right)$ for some scattered compact space $K$. Here $\left(B_{C(K)^{*}}, w^{*}\right)$ denotes the unit ball in $C(K)^{*}$ equipped with the weak* topology.

Proof. Assume that $K$ is a scattered compact space. Let $T_{n}: C\left(K^{n}\right) \rightarrow$ $C\left(B_{C(K)^{*}}\right)$ be given by $T_{n}(f)(\mu)=2^{-n} \int f d \mu^{n}$ where $\mu^{n} \in B_{C\left(K^{n}\right)^{*}}$ is the product measure.

Define $T:\left(C(K) \times C\left(K^{2}\right) \times C\left(K^{3}\right) \times \cdots\right)_{c_{0}} \rightarrow C\left(B_{C(K)^{*}}\right)$ by $T\left(f_{1}, f_{2}, \ldots\right)$ $=\sum_{n=1}^{\infty} T_{n}\left(f_{n}\right)$. By the Stone-Weierstrass theorem the image of $T$ is dense. Indeed it separates points since the image of $T_{1}$ does. To see that it is a subalgebra it is enough to notice that $T_{n}\left(f_{n}\right) T_{m}\left(f_{m}\right)=T_{n+m}\left(f_{n} \times f_{m}\right)$. Because $K^{n}$ is scattered for all $n \in \mathbb{N}$, by Fact 3.5 we have $B_{C(K)^{*}} \in \mathcal{G}$. Thus if $L \subset B_{C(K)^{*}}$ then $L \in \mathcal{G}$. On the other hand if $L \in \mathcal{G}$ then by a duality argument $L \subset B_{C(L) *} \subset B_{C(K) *}$ for some scattered compactum $K$.

Arvanitakis 3] considers a similar class (a subclass of $\mathcal{G}$ ) of compact spaces. More precisely he considers all compacta that can be embedded into the compact space $P(K)$ of all probability measures on a scattered compact space $K$ endowed with the weak* topology. He shows that all almost totally disconnected compact spaces are embeddable in $P(K)$ for some scattered compact space $K$, hence by Fact 3.8 the almost totally disconnected spaces constitute a subclass of $\mathcal{G}$.

Corollary 3.9. The classes of compact spaces defined in Definition 3.7 are subclasses of $\mathcal{G}$.

However it is of independent interest that the class of uniformly Eberlein compacta can be characterized in the spirit of Definition 3.6. More precisely, $L$ is an Eberlein compactum if $C(L)$ is generated by the simplest possible $C(K)$ space where $K$ is scattered.

THEOREM 3.10. Let $L$ be a compact space. The following conditions are equivalent:

(i) L is uniformly Eberlein,

(ii) $C(L)$ is Hilbert generated,

(iii) $C(L)$ is generated by $c_{0}(\Gamma)$ for some nonempty set $\Gamma$.

Proof. Conditions (i) and (ii) are equivalent (Theorem 2 of [11]). Since the natural embedding shows that $\ell_{2}(\Gamma)$ generates $c_{0}(\Gamma)$ the proof will be finished once we show that $C(L)$ is generated by some $c_{0}(\Gamma)$ if $L$ is a uniformly Eberlein compact space. Note that according to Fact 3.4 no nonseparable Hilbert space is generated by $c_{0}(\Gamma)$ (or even scattered generated). 
Let us denote by $A(\Gamma)$ the one-point compactification of a discrete set $\Gamma$. From Theorem 4 of [4] we know that for every uniformly Eberlein compact space $L$ there exists a closed subset $M$ of the space $A(\Gamma)^{\omega}$ and an onto map $\varphi: M \rightarrow L$ admitting a regular averaging operator. Hence there exists an operator from $C(M)$ onto $C(L)$, and by taking the restriction operator from $C\left(A(\Gamma)^{\omega}\right)$ onto $C(M)$ we arrive at the conclusion that $C(L)$ is generated by $C\left(A(\Gamma)^{\omega}\right)$. Let $T:\left(C(A(\Gamma)) \times C\left(A(\Gamma)^{2}\right) \times \cdots\right)_{c_{0}} \rightarrow C\left(A(\Gamma)^{\omega}\right)$ be defined by

$$
T\left(\left(f_{1}, f_{2}, \ldots\right)\right)=\sum_{n=1}^{\infty} \frac{1}{2^{n}} f_{n} \circ \pi_{n}
$$

where $\pi_{n}: A(\Gamma)^{\omega} \rightarrow A(\Gamma)^{n}$ is the natural projection onto the first $n$ coordinates. From the Stone-Weierstrass theorem we clearly see that the image of $T$ is dense in $C\left(A(\Gamma)^{\omega}\right)$. As for every $n$ there exists an isomorphism $\Lambda_{n}: c_{0}(\Gamma) \rightarrow C\left(A(\Gamma)^{n}\right)$ (see Theorem 1.1 of [15]), the operator $\Lambda: c_{0}(\Gamma)=\left(c_{0}(\Gamma) \times c_{0}(\Gamma) \times \cdots\right)_{c_{0}} \rightarrow\left(C(A(\Gamma)) \times C\left(A(\Gamma)^{2}\right) \times \cdots\right)_{c_{0}}$ defined by

$$
\Lambda\left(f_{1}, f_{2}, \ldots\right)=\left(\frac{1}{\left\|\Lambda_{1}\right\|} \Lambda_{1} f_{1}, \frac{1}{2\left\|\Lambda_{2}\right\|} \Lambda_{2} f_{2}, \ldots\right)
$$

has a dense range in $\left(C(A(\Gamma)) \times C\left(A(\Gamma)^{2}\right) \times \cdots\right)_{c_{0}}$. The operator $T \circ \Lambda$ shows that $C\left(A(\Gamma)^{\omega}\right)$ is generated by $c_{0}(\Gamma)$, hence also $C(L)$ is generated by $c_{0}(\Gamma)$.

Let us remark that reasoning as above one can easily prove that the class $\mathcal{G}$ is closed under countable products.

In order to obtain an upper estimate for the class $\mathcal{G}$ we need the following:

Theorem 3.11 (Stegall [18]). Let $L$ be a compact space. Then $L \in \mathbf{R N}$ (i.e. $L$ is a Radon-Nikodym compactum) if and only if $C(L)$ is Asplund generated. In particular $\mathcal{G} \subset \mathbf{R N}$ (see Fact 2.3).

Let us recall the definition of the class $\mathbf{R N}$ :

Definition 3.12. Let $L$ be a compact space. We say that $L \in \mathbf{R N}$ if $L$ is homeomorphic to a weak* compact subset of the dual of an Asplund space.

However the following problem remains open:

Problem 3.13. Is $\mathcal{G}=\mathbf{R N}$ ?

A similar problem was posed by Argyros (see [3]):

Problem 3.14. Is every Radon-Nikodym compactum embeddable into a compact space $P(K)$ for some scattered compact space $K$ ?

Obviously a positive answer to the above question would also give a positive answer to Problem 3.13 (see Theorem 3.8). Both problems are also 
recalled in the survey paper of Avilés and Kalenda [5]. On the other hand it is of interest whether Problems 3.13 and 3.14 are equivalent. More precisely:

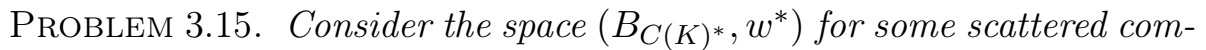
pact space $K$. Is $\left(B_{C(K)^{*}}, w^{*}\right)$ homeomorphic to a subspace of $P(L)$ for some scattered compact $L$ ?

Obviously if the answer is negative the counterexample would give an answer to Problem 3.14.

Acknowledgments. I am very grateful to the anonymous referee for suggestions which improved the clarity of this article.

The author was partially supported by KBN grant 2 P03 A 02524 during his stay at the Institute of Mathematics, Polish Academy of Sciences.

\section{References}

[1] D. Amir and J. Lindenstrauss, The structure of weakly compact sets in Banach spaces, Ann. of Math. 88 (1968), 35-46.

[2] N. Aronszajn, Differentiability of Lipschitzian mappings between Banach spaces, Studia Math. 57 (1976), 147-190.

[3] A. Arvanitakis, Some remarks on Radon-Nikodym compact spaces, ibid. 172 (2002), 41-60.

[4] A. Avilés, Countable products of spaces of finite sets, ibid. 151 (2002), 147-159.

[5] A. Avilés and O. Kalenda, Compactness in Banach space theory - selected problems, Rev. R. Acad. Cien. Ser. A. Mat. 104 (2010), 337-352.

[6] Y. Benyamini and J. Lindenstrauss, Geometric Nonlinear Functional Analysis, Colloq. Publ. 48, Amer. Math. Soc., 1993.

[7] J. M. Borwein and W. B. Moors, Separable determination of integrability and minimality of the Clarke subdifferential mapping, Proc. Amer. Math. Soc. 128 (1999), $215-221$.

[8] J. P. R. Christensen, Measure theoretic zero sets in infinite dimensional spaces and applications to differentiability of Lipschitz mappings, in: Actes du Deuxième Colloque d'Analyse Fonctionnelle de Bordeaux (Bordeaux, 1973), I, Publ. Dép. Math. (Lyon) 10 (1973), no. 2, 29-39.

[9] R. Deville, G. Godefroy and V. Zizler, Smoothness and Renormings in Banach Spaces, Pitman Monogr. Surveys Pure Appl. Math. 64, Longman Sci. \& Tech., Harlow, 1993.

[10] M. Fabian, Gâteaux Differentiability of Convex Functions and Topology. Weak Asplund Spaces, Canad. Math. Soc. Ser. Monogr. Adv. Texts, Wiley, New York, 1997.

[11] M. Fabian, G. Godefroy and V. Zizler, The structure of uniformly Gâteaux smooth Banach spaces, Israel J. Math. 124 (2001), 243-252.

[12] W. Holsztyński, Continuous mappings induced by isometries of spaces of continuous functions, Studia Math. 26 (1966), 133-136.

[13] J. Lindenstrauss and D. Preiss, On Fréchet differentiability of Lipschitz maps betwen Banach spaces, Ann. of Math. 157 (2003), 257-288.

[14] P. Mankiewicz, On the differentiability of Lipschitz mappings in Fréchet spaces, Studia Math. 45 (1973), 15-29. 
[15] W. Marciszewski, On Banach spaces $C(K)$ isomorphic to $c_{0}(\Gamma)$, ibid. 156 (2003), 295-302.

[16] R. R. Phelps, Convex Functions, Monotone Operators and Differentiability, 2nd ed,. Lecture Notes in Math. 1364, Springer, 1993.

[17] D. Preiss, Gâteaux differentiable functions are somewhere Fréchet differentiable, Rend. Circ. Mat. Palermo 33 (1984), 122-133.

[18] Ch. Stegall, The Radon-Nikodym property in conjugate Banach spaces II, Trans. Amer. Math. Soc. 264 (1981), 507-519.

Rafał Górak

Technical University of Warsaw

Pl. Politechniki 1

00-661 Warszawa, Poland

E-mail: rafalgorak@gmail.com

Received December 14, 2010;

received in final form January 6, 2011 